Comment. Math. Helv. 74 (1999) 179-200

(C) 1999 Birkhäuser Verlag, Basel

\title{
The conjugacy problem for Dehn twist automorphisms of free groups
}

\author{
Marshall M. Cohen ${ }^{1}$ and Martin Lustig
}

\begin{abstract}
A Dehn twist automorphism of a group $G$ is an automorphism which can be given (as specified below) in terms of a graph-of-groups decomposition of $G$ with infinite cyclic edge groups. The classic example is that of an automorphism of the fundamental group of a surface which is induced by a Dehn twist homeomorphism of the surface. For $G=F_{n}$, a non-abelian free group of finite rank $n$, a normal form for Dehn twist is developed, and it is shown that this can be used to solve the conjugacy problem for Dehn twist automorphisms of $F_{n}$.
\end{abstract}

Mathematics Subject Classification (1991). 20E, 57M, $57 \mathrm{~N}$.

Keywords. Free group, Dehn twist automorphism, normal form, algorithm, conjugacy problem, graph of groups decomposition, centralizer, index, fixed subgroup.

\section{Introduction}

If $\phi_{1}$ and $\phi_{2}$ are automorphisms of groups $G_{1}$ and $G_{2}$ respectively, then we say that $\phi_{1}$ and $\phi_{2}$ are conjugate if there is an isomorphism $\alpha: G_{1} \longrightarrow G_{2}$ such that $\phi_{2}=\alpha \phi_{1} \alpha^{-1}$. They are conjugate up to inner automorphism if there is an isomorphism $\alpha: G_{1} \longrightarrow G_{2}$ and an element $x \in G_{2}$ such that $\phi_{2}=a d_{x} \alpha \phi_{1} \alpha^{-1}$. (Here $a d_{x}$ denotes the inner automorphism of $G_{2}$ given by $a d_{x}(g)=x g x^{-1}$ for all $g \in G_{2}$.) If $G_{1}=G_{2}=G$, then $\phi_{1}$ and $\phi_{2}$ are conjugate up to inner automorphism precisely when they represent conjugate elements $\widehat{\phi_{1}}, \widehat{\phi_{2}}$ of the outer automorphism group $\operatorname{Out}(G)$.

This paper is concerned with the determination of whether two given Dehn twist automorphisms (defined below) of the free group $F_{n}$ are conjugate or conjugate up to inner automorphism. The results here will be extended in the forthcoming paper $[\mathrm{KLV}]$ to roots of Dehn twist automorphisms (and hence to all au-

\footnotetext{
1 The first author was supported by a travel grant from the Deutsche Forschungsgemeinschaft (DFG). The second author was supported by a Heisenberg Stipendium from the DFG and did part of this work as a member of the Institute for Advanced Study in Princeton, N. J. during Spring 1995.
} 
tomorphisms of $F_{n}$ of linear growth) and will be a key part of the second author's complete solution of the conjugacy problem for $\operatorname{Out}\left(F_{n}\right)$ as announced in [L1].

A Dehn twist $D=D\left(\mathcal{G},\left(z_{e}\right)_{e \in E(\mathcal{G})}\right)$ consists of a graph of groups $\mathcal{G}$ and, for every edge $e$ of $\mathcal{G}$, a specified twistor $z_{e}$ in the center of the edge group $G_{e}$. (See 5.) Every Dehn twist determines a Dehn twist automorphism $D_{v}$ of the fundamental group $\pi_{1}(\mathcal{G}, v)$ for each vertex $\mathrm{v}$ of $\mathcal{G}$ and hence an automorphism of the abstract group $\pi_{1}(\mathcal{G})$ which is well defined up to inner automorphism. Thus $D$ determines an outer automorphism $\widehat{D} \in \operatorname{Out}\left(\pi_{1}(\mathcal{G})\right)$.

A Dehn twist automorphism of $F_{n}$ is an automorphism which is conjugate to such a Dehn twist automorphism $D_{v}$ of $\pi_{1}(\mathcal{G}, v)$ for some graph of groups $\mathcal{G}$. Dehn twists on $F_{n}$ are the natural analogues of geometric Dehn twists (i.e. multiple Dehn twists along sets of disjoint simple closed curves on surfaces); indeed, the automorphisms induced by the geometric Dehn twists on surfaces with boundary are special cases of Dehn twist automorphisms of $F_{n}$. On the other hand, an example of a Dehn twist automorphism of $F(a, b, c)$ which is not geometric is given by the automorphism $a \rightarrow a, b \rightarrow b, c \rightarrow w c w^{-1}$, if $w \in F(a, b)$ is not a power of $x, x y x^{-1} y^{-1}$, or $x^{2} y^{2}$ for any basis $x, y$ of $F(a, b)$.

Dehn twists can be given in clearly inefficient ways. We define efficient Dehn twists in 6. The main result of this paper is the following classification of automorphisms determined by efficient Dehn twists. (For background on graph of groups isomorphisms and the induced isomorphisms of their fundamental groups, see 4.)

1.1. Theorem. Suppose that $\mathcal{G}_{1}$ and $\mathcal{G}_{2}$ are graphs of groups with $\pi_{1}\left(\mathcal{G}_{1}\right) \cong$ $\pi_{1}\left(\mathcal{G}_{2}\right) \cong F_{n}$ and that $v$ and $w$ are vertices of $\mathcal{G}_{1}$ and $\mathcal{G}_{2}$ respectively. Let $D_{1}=D\left(\mathcal{G}_{1},\left(z_{e}\right)_{e \in E\left(\mathcal{G}_{1}\right)}\right)$ and $D_{2}=D\left(\mathcal{G}_{2},\left(z_{e}\right)_{e \in E\left(\mathcal{G}_{2}\right)}\right)$ be efficient Dehn twists inducing automorphisms $D_{v}$ and $D_{w}$ of $\pi_{1}\left(\mathcal{G}_{1}, v\right)$ and $\pi_{1}\left(\mathcal{G}_{2}, w\right)$ respectively. Let $h: \pi_{1}\left(\mathcal{G}_{1}, v\right) \longrightarrow \pi_{1}\left(\mathcal{G}_{2}, w\right)$ be an isomorphism.

(a) $\widehat{D_{2}}=h{\widehat{D_{1} h^{-1}}}^{-1} \operatorname{Out}\left(\pi_{1}\left(\mathcal{G}_{2}\right)\right)$ if and only if there is a graph of groups isomorphism $H: \mathcal{G}_{1} \longrightarrow \mathcal{G}_{2}$ which induces the isomorphism $h$ up to inner automorphism (i.e., $\widehat{H}=\widehat{h}$ ) and which takes twistors to twistors (i.e., $H_{e}\left(z_{e}\right)=z_{H(e)}$ for all $e \in$ $E\left(\mathcal{G}_{1}\right)$ ).

(b) $D_{w}=h D_{v} h^{-1} \in \operatorname{Aut}\left(\pi_{1}\left(\mathcal{G}_{2}, w\right)\right)$ if and only if there is a graph of groups isomorphism $H: \mathcal{G}_{1} \longrightarrow \mathcal{G}_{2}$ which takes $v$ to $w$, with induced isomorphism $H_{* v}=$ $h: \pi_{1}\left(\mathcal{G}_{1}, v\right) \longrightarrow \pi_{1}\left(\mathcal{G}_{2}, w\right)$, and which takes twistors to twistors.

The material developed to prove Theorem 1.1 allows us to determine along the way the centralizer, fixed subgroup, index and infinite attracting fixed words (there aren't any) of a Dehn twist automorphism of a free group. These results are given in 7 .

In 8. we give an algorithm for transforming an arbitrary Dehn twist $D$ of a graph of groups $\mathcal{G}$ to an efficient Dehn twist $D^{\prime}$ of a graph of groups $\mathcal{G}^{\prime}$ in such a way that the induced isomorphisms of $\pi_{1}(\mathcal{G})$ and $\pi_{1}\left(\mathcal{G}^{\prime}\right)$ are conjugate up to inner automorphism. We further point out that one can use the Whitehead algorithm to 
decide whether two graphs of groups are isomorphic in such a way as to preserve the data in part (a) or (b) of Theorem 1.1. This leads to our solution of the conjugacy problem for Dehn twist outer automorphisms:

1.2. Theorem. There exists an algorithm which, given two Dehn twists $D_{1}$ and $D_{2}$ based on graphs of groups $\mathcal{G}_{1}$ and $\mathcal{G}_{2}$ with $\pi_{1}\left(\mathcal{G}_{1}\right) \cong \pi_{1}\left(\mathcal{G}_{2}\right) \cong F_{n}$, decides in finitely many steps whether the induced outer automorphisms $\widehat{D_{1}}$ and $\widehat{D_{2}}$ are conjugate. If $D_{1}$ and $D_{2}$ are efficient and if $v$ and $w$ are vertices of the graph of $\mathcal{G}_{1}$ and the graph of $\mathcal{G}_{2}$ respectively, this algorithm also decides whether the induced automorphisms $D_{v}$ and $D_{w}$ of $\pi_{1}\left(\mathcal{G}_{1}, v\right)$ and $\pi_{1}\left(\mathcal{G}_{2}, w\right)$ are conjugate.

Remarks: (1) This paper is written totally within the genre of graphs of groups and their associated actions on R-trees. If an automorphism $\varphi \in \operatorname{Aut}\left(F_{n}\right)$ is given in terms of the image of some basis of $F_{n}$, it is possible [L2] to decide whether $\varphi$ is a Dehn twist automorphism - whether it is conjugate to some automorphism $D_{v}$ of $\pi_{1}(\mathcal{G}, v)$ given by a Dehn twist $D$ of a graph of groups $\mathcal{G}$ - and, if so, to derive from the data for $\varphi$ the data for $D$ and for $\mathcal{G}$, namely $D=D\left(\mathcal{G},\left(z_{e}\right)_{e \in E(\mathcal{G})}\right)$. However, in this paper - and in particular in Theorem 1.2 - we always assume that a Dehn twist automorphism is given in terms of the graph of groups data (see 8.1).

(2) The algorithm in Theorem 1.2 is purely combinatorial and operates entirely in terms of the graph of groups data of $D_{1}$ and $D_{2}$. It is noteworthy (see 6.7) that the underlying justification of this combinatorial algorithm comes from the study of the dynamics of Dehn twist automorphisms acting on the closure of CullerVogtmann's "Outer Space"; we use a result of our previous paper [CL2] concerning these dynamics to prove Theorem 1.1, which in turn implies Theorem 1.2.

\section{Outer homomorphisms of groups}

Much of this paper concerns outer automorphisms rather than ordinary automorphisms. In this context, the following notion turns out to be natural and useful:

2.1. Definition. Let $f: G \rightarrow H$ be a group homomorphism. Then we denote by $\widehat{f}: G \rightarrow H$ the outer homomorphism induced by $f$. This is the equivalence class

$$
\hat{f}=\left\{a d_{h} f: G \rightarrow H \mid h \in H\right\}
$$

of homomorphisms from $G$ to $H$.

Notice that for any homomorphisms $f_{1}: G_{1} \rightarrow G_{2}$ and $f_{2}: G_{2} \rightarrow G_{3}$ one has $\widehat{f_{2} f_{1}}=\widehat{f_{2}} \widehat{f_{1}}$. Furthermore, for any automorphism $f: G \rightarrow G$ the set $\hat{f}=\operatorname{Inn}(G) \cdot f$ is precisely the induced outer automorphism in the usual sense. The notion of outer homomorphism, though it does not seem to be standard, is the natural morphism 
induced on the level of fundamental groups by continuous maps between pathconnected topological spaces without specified base point.

If $\alpha: G \rightarrow G^{\prime}$ and $\beta: H \rightarrow H^{\prime}$ are fixed isomorphisms, then every $\hat{f}: G \rightarrow H$ induces $\widehat{f^{\prime}}=\beta \widehat{f \alpha^{-1}}: G^{\prime} \rightarrow H^{\prime}$, and $\widehat{f}^{\prime}$ does not depend on $\alpha$ and $\beta$ but only on their induced outer isomorphisms. This observation can be applied as follows to malnormal subgroups. (Recall that $U \subset G$ is a malnormal subgroup if $g \in G$ and $g \notin U$ implies that $\left.g U g^{-1} \cap U=\{1\}\right)$.

2.2. Lemma. Let $U_{i} \subset G$ and $V_{i} \subset H(i=1,2)$ be malnormal subgroups of $G$ and $H$ with $U_{1}$ conjugate to $U_{2}$ and $V_{1}$ conjugate to $V_{2}$; say $U_{2}=g U_{1} g^{-1}$ and $V_{2}=h V_{1} h^{-1}$ for some $g \in G$ and $h \in H$.

(a) Every outer homomorphism $\widehat{f}_{1}: U_{1} \rightarrow V_{1}$ with representative $f_{1}$ determines an outer homomorphism $\widehat{F_{1}}: U_{2} \rightarrow V_{2}$ with representative $F_{1}=a d_{h} \circ f_{1} \circ a d_{g}{ }^{-1}$. This outer homomorphism is independent of the choice of representative $f_{1}$ and of the conjugators $g$ and $h$. In particular, if $U_{1}$ and $U_{2}$ are conjugate malnormal subgroups of a group $G$ then there is a canonical identification between $\operatorname{Out}\left(U_{1}\right)$ and $\operatorname{Out}\left(U_{2}\right)$.

(b) Let $f: G \longrightarrow H$ be a homomorphism with $f\left(U_{1}\right) \subset V_{1}$ and $f\left(U_{2}\right) \subset V_{2}$. Let $f_{i}: U_{i} \longrightarrow V_{i}(i=1,2)$ be the maps induced by restricting $f$. Then $\widehat{f_{2}}=\widehat{F_{1}}$ where $F_{1}=a d_{h} \circ f_{1} \circ a d_{g}^{-1}$, as in (a).

Proof. (a) We first note that, if $x \in V_{1}$ then the outer homomorphism class of $\operatorname{ad}_{h} f_{1} \operatorname{ad}_{g}^{-1}$ equals that of $\operatorname{ad}_{h} \operatorname{ad}_{x} f_{1} \operatorname{ad}_{g}^{-1}$. This is because $h x h^{-1} \in V_{2}$ and

$$
\operatorname{ad}_{h} \operatorname{ad}_{x} f_{1} \operatorname{ad}_{g}^{-1}=\operatorname{ad}_{h x h^{-1}}\left(\operatorname{ad}_{h} f_{1} \operatorname{ad}_{g^{-1}}\right) .
$$

Now suppose that $g^{\prime} \in G, h^{\prime} \in H$ are elements such that $U_{2}=g^{\prime} U_{1} g^{-1}$ and $V_{2}=h^{\prime} V_{1} h^{\prime-1}$. Then the outer homomorphism class of $\operatorname{ad}_{h} f_{1} \operatorname{ad}_{g}^{-1}$ equals that of $\operatorname{ad}_{h^{\prime}} f_{1} \operatorname{ad}_{g^{\prime}}^{-1}$ because malnormality implies that $g^{-1} g^{\prime} \in U_{1}$ and $h^{-1} h^{\prime} \in V_{1}$ and because

$$
a d_{h^{\prime}} f_{1} \cdot \operatorname{ad}_{g^{\prime}}^{-1}=\operatorname{ad}_{h} \operatorname{ad}_{\left(h^{-1} h^{\prime}\right)} f_{1} \operatorname{ad}_{g^{\prime-1} g} \operatorname{ad}_{g^{-1}}=\operatorname{ad}_{h} \operatorname{ad}_{\left(h^{-1} h^{\prime}\right) \cdot f_{1}\left(g^{-1} g^{\prime}\right)} f_{1} \operatorname{ad}_{g^{-1}}
$$

Since $\left(h^{-1} h^{\prime}\right) \cdot f_{1}\left(g^{-1} g^{\prime}\right) \in V_{1}$ the first paragraph of the proof applies.

The preceding two paragraphs prove (a).

(b) We have $f=a d_{f(g)} f a d_{g^{-1}}$ and since $U_{2}=g U_{1} g^{-1}$ we can write this as $f_{2}=a d_{f(g)} f_{1} a d_{g^{-1}} \mid U_{2}$. If $f\left(U_{1}\right)=\{1\}$ the result claimed is trivial. In case $f\left(U_{1}\right) \neq\{1\}$, the given element $h$ with $h^{-1} V_{2} h=V_{1}$ satisfies

$$
f(g) h^{-1}\left(h f\left(U_{1}\right) h^{-1}\right) h f(g)^{-1}=f\left(U_{2}\right)
$$

and hence

$$
f(g) h^{-1} V_{2} h f(g)^{-1} \cap V_{2} \neq\{1\} .
$$


Thus malnormality implies that $x \equiv f(g) h^{-1} \in V_{2}$ so that $f_{2}=a d_{x} a d_{h} f_{1} a d_{g^{-1}}$ $=a d_{x} F_{1}$ with $x \in V_{2}$. Thus $\widehat{f_{2}}=\widehat{F_{1}}$ as claimed.

\section{Graphs of groups}

For the convenience of the reader we recall in this and the following section some standard definitions and facts concerning graphs of groups. For general background see $[\mathrm{S}],[\mathrm{B}],[\mathrm{C}]$ or $[\mathrm{D}-\mathrm{D}]$. We mainly follow the notation of [CL2]. We include some basic results which we will need which do not seem to have appeared before. (See (3.9) for normal forms for representatives of conjugacy classes and (3.10) for the fact that vertex groups are malnormal in path groups.)

3.1. A graph of groups is given by

$$
\mathcal{G}=\left(\Gamma(\mathcal{G}),\left\{G_{v}\right\}_{v \in V(\mathcal{G})},\left\{G_{e}\right\}_{e \in E(\mathcal{G})},\left\{f_{e}: G_{e} \rightarrow G_{\tau(e)}\right\}_{e \in E(\mathcal{G})}\right)
$$

where we use the following notation:

$-\Gamma(\mathcal{G})$ is a finite connected graph,

$-V(\mathcal{G})$ is the set of vertices of $\Gamma(\mathcal{G})$,

$-E(\mathcal{G})$ is the set of oriented edges of $\Gamma(\mathcal{G})$.

For any $v \in V(\mathcal{G})$ and $e \in E(\mathcal{G})$ we denote:

$-\bar{e}$ is the edge oppositely oriented to $e$,

$-\tau(e)$ is the terminal vertex of $e$ (so that $\tau(\bar{e})$ is the initial vertex of $e$ ),

- $G_{v}$ is the vertex group at $v$,

- $G_{e}=G_{\bar{e}}$ is the edge group at $e$,

$-f_{e}: G_{e} \rightarrow G_{\tau(e)}$ is an injective homomorphism.

3.2. We denote by $\Pi(\mathcal{G})$ the path group of $\mathcal{G}$ (called the Bass group $\beta(\mathcal{G})$ in [CL2]), which is generated by the stable letters $t_{e}(e \in E(\mathcal{G}))$ and the elements $r \in G_{v}(v \in V(\mathcal{G}))$, subject to the relations in the $G_{v}$, and to

(i) $t_{\bar{e}}=t_{e}^{-1} \quad$ and

(ii) $t_{e} f_{e}(a) t_{e}^{-1}=f_{\bar{e}}(a) \in G_{\tau(\bar{e})} \quad$ for all $a \in G_{e}, e \in E(\mathcal{G})$.

3.3. Every element of $\Pi(\mathcal{G})$ is given by a word

$$
W=r_{0} t_{1} r_{1} \ldots t_{q} r_{q}
$$

where $t_{i}=t_{e_{i}}$ and each $r_{i}$ is an element of the free product $*\left\{G_{v} \mid v \in V(\mathcal{G})\right\}$. We say that $W$ is connected, with initial vertex $\tau\left(\bar{e}_{1}\right)$ and terminal vertex $\tau\left(e_{1}\right)$, if $r_{0} \in G_{\tau\left(\bar{e}_{q}\right)} r_{q} \in G_{\tau\left(e_{q}\right)}$ and $\tau\left(e_{i}\right)=\tau\left(\bar{e}_{i+1}\right)$ with $r_{i} \in G_{\tau\left(e_{i}\right)}$ for all $i=1, \ldots, q-1$. Note that connected words are transformed to connected words when they are shortened by the operations of 3.2, but that a trivial word $f_{\bar{e}}(a)^{-1} t_{e} f_{e}(a) t_{\bar{e}}$ can be inserted into a connected word so as to make it non-connected. Finally, $W$ is 
a closed, connected word based at $v$ if it is connected and $v=\tau\left(\bar{e}_{1}\right)=\tau\left(e_{q}\right)$. This includes all $W \in G_{v}$.

3.4. We denote by $\pi_{1}(\mathcal{G}, v) \subset \Pi(\mathcal{G})$ the fundamental group of $\mathcal{G}$ based at the vertex $v$. It consists precisely of those elements of $\Pi(\mathcal{G})$ which are represented by a closed connected word based at $v$. For distinct vertices $v_{1}, v_{2} \in V(\mathcal{G})$ the subgroups $\pi_{1}\left(\mathcal{G}, v_{1}\right)$ and $\pi_{1}\left(\mathcal{G}, v_{2}\right)$ are conjugate in $\Pi(\mathcal{G})$. Notice that $\Pi(\mathcal{G})$ is canonically isomorphic to $\pi_{1}\left(\mathcal{G}_{*}, v_{*}\right)=\Pi\left(\mathcal{G}_{*}\right)$ where $\mathcal{G}_{*}$ is the graph of groups obtained from $\mathcal{G}$ by identifying all vertices $v \in V(\mathcal{G})$ to a unique vertex $v_{*}$ and defining its vertex group to be the free product of all $G_{v}$ (and replacing each $f_{e}$ by [inclusion $\circ f_{e}$ ]).

3.5. A word $W \in \Pi(\mathcal{G})$ as in 3.3 is reduced if $q=0$ or if $t_{i}=t_{i+1}^{-1}$ implies that $r_{i} \notin f_{e_{i}}\left(G_{e_{i}}\right)(i=1, \ldots, q-1)$. A non-trivial reduced word need not be connected, but can be identified with a (necessarily connected) reduced word in $\Pi\left(\mathcal{G}_{*}\right)$. So classical results of Bass and Serre on connected words in a path group apply to arbitrary words in $\Pi(\mathcal{G})$. By applying the relations 3.2 above sufficiently often any word $W \in \Pi(\mathcal{G})$ can be transformed into a reduced word. Also, reduced words have the following uniqueness property:

3.6. Proposition. If $V=r_{0} t_{1} r_{1} \ldots t_{q} r_{q}$ and $W=s_{0} t_{1}^{\prime} s_{1} \ldots t_{q^{\prime}}^{\prime} s_{q^{\prime}}$ are reduced words representing the same element $g \in \Pi(\mathcal{G})$ then:

(a) $t_{i}=t_{i}^{\prime}$ for all $i=1, \ldots, q$. In particular, $q=q^{\prime}$ ( $\equiv$ length $(g)$ ).

(b) For all $i=1, \ldots, q$ there exist elements $h_{i} \in G_{e_{i}}$ such that

$$
\begin{aligned}
s_{0} & =r_{0} f_{\bar{e}_{1}}\left(h_{1}^{-1}\right), \\
s_{i} & =f_{e_{i}}\left(h_{i}\right) r_{i} f_{\bar{e}_{i}+1}\left(h_{i+1}^{-1}\right) \quad \text { for } \quad i=1, \ldots, q-1, \text { and } \\
s_{q} & =f_{e_{q}}\left(h_{q}\right) r_{q} .
\end{aligned}
$$

(c) $V$ is connected if and only if $W$ is connected.

Proof. (a) and (b) follow from [S, p. 50] or [B, 1.10], while (c) follows from (a) and (b).

3.7. (a) A product $V W$ of two reduced words in $\Pi(\mathcal{G})$ is called reduced if the concatenation of the two words is reduced. This is equivalent to "length $(V W)=$ length $(V)+\operatorname{length}(W)$ ", and in this case we write $V * W$ for $V W$. For example, if length $(V)=0$, then $V W=V * W$ for any $W$. One must be careful concerning connectivity. A reduced connected word $V$ may be factored as the reduced product of reduced non-connected words: $V=A * B=(A * r) *\left(r^{-1} * B\right)$, where $r \in G_{v}$ for some vertex $v$ far away from the path carrying $V=A * B$. However, we do have:

(b) If $V=A * B * C$ with $V$ and $B$ connected, then $A$ and $C$ are also connected and the terminal vertex of $A$ equals the initial vertex of $B$ and the terminal vertex of $B$ equals the intial vertex of $C$. 
3.8. A word $W \in \Pi(\mathcal{G})$ is cyclically reduced if it is reduced and if, furthermore, $\tau\left(e_{q}\right)=\tau\left(\bar{e}_{1}\right)$ and $t_{1}=t_{q}^{-1}$ imply $r_{q} r_{0} \notin f_{e_{q}}\left(G_{e_{q}}\right)$. Through cyclic permutations (which can be effected by conjugation in $\Pi(\mathcal{G})$ ) and the relations of 3.2 one can transform any word $W$ into a cyclically reduced word.

3.9. Proposition. Closed, connected, cyclically reduced words $V$ and $W$ represent conjugate elements of $\Pi(\mathcal{G})$ if and only if there is a cyclic permutation $W_{2} * W_{1}$ of $W=W_{1} * W_{2}$ and an element $r \in G_{v}$ (where $V$ is based at $v \in V(\mathcal{G})$ ) such that $V=r * W_{2} * W_{1} * r^{-1}$.

Proof. If there exists such a cyclic permutation then clearly the elements are conjugate. Now suppose that $V$ and $W$ represent conjugate elements.

Let $U \in \Pi(\mathcal{G})$ be a reduced word with $V=U W U^{-1}$. As $W$ is cyclically reduced, one has either $U W=U * W$ or $W U^{-1}=W * U^{-1}$. We assume the first case (the second works similarly) and obtain

$$
\begin{aligned}
& \operatorname{length}(V)=\operatorname{length}\left(U W U^{-1}\right) \geq \operatorname{length}(U W)-\operatorname{length}\left(U^{-1}\right)= \\
& \operatorname{length}(U)+\operatorname{length}(W)-\operatorname{length}\left(U^{-1}\right)=\operatorname{length}(W) .
\end{aligned}
$$

By symmetry between $V$ and $W$ we get length $(V)=$ length $(W)$. We can assume w.l.g. that $U$ is not a reduced product $U=U^{\prime} * W^{k}$ for any $k \geq 1$. Thus $U^{-1}$ will have all stable letters cancelled against $W$ when reducing the product $U W U^{-1}$. It follows that $W$ is a reduced product of connected subwords $W=W_{1} * W_{2}$ with length $\left(W_{2} U^{-1}\right)=0$. Since $W_{1}$ and $V=U * W_{1} *\left(W_{2} U^{-1}\right)$ are connected, it follows by $3.7(\mathrm{~b})$ that $W_{2} U^{-1}$ is connected. Then $W_{2} U^{-1} \equiv r \in G_{v}$ where $v$ is the terminal vertex of $W_{1}$ and the initial vertex of $W_{2}$. Hence $V=U W U^{-1}=$ $\left(U W_{2}^{-1}\right) * W_{2} * W_{1} *\left(U W_{2}^{-1}\right)^{-1}=r * W_{2} * W_{1} * r^{-1}$, as claimed.

3.10. Lemma. The subgroup $\pi_{1}(\mathcal{G}, v)$ is malnormal in $\Pi(\mathcal{G})$.

Proof. Let $V, W \in \pi_{1}(\mathcal{G}, v)$ and $U \in \Pi(\mathcal{G})$ be reduced words with $V=U W U^{-1}$. Since malnormality of subgroups is invariant with respect to conjugation, we can assume that $W$ is cyclically reduced. Following the proof of 3.9 we may write $W=W_{1} * W_{2}$ and $V=U * W_{1} *\left(W_{2} U^{-1}\right)$ where $W_{1}$ is connected. It follows from 3.7 (b) that $U$ is a connected word which begins and ends at $v$. Thus $U \in \pi_{1}(\mathcal{G}, v)$.

3.11. The malnormality given by 3.10 , combined with Lemma 2.2 , tells us that for any graphs of groups $\mathcal{G}_{1}, \mathcal{G}_{2}$ and vertices $v, v^{\prime} \in V\left(\mathcal{G}_{1}\right)$ and $w, w^{\prime} \in V\left(\mathcal{G}_{2}\right)$ there is a canonical identification between the outer homomorphisms from $\pi_{1}\left(\mathcal{G}_{1}, v\right)$ to $\pi_{1}\left(\mathcal{G}_{2}, w\right)$ and those from $\pi_{1}\left(\mathcal{G}_{1}, v^{\prime}\right)$ to $\pi_{1}\left(\mathcal{G}_{2}, w^{\prime}\right)$. To be precise, $\pi_{1}\left(\mathcal{G}_{1}, v^{\prime}\right)=$ $\operatorname{ad}_{W}\left(\pi_{1}\left(\mathcal{G}_{1}, v\right)\right)$ if and only if $W \in \Pi\left(\mathcal{G}_{1}\right)$ is a connected word with initial vertex $v^{\prime}$ and terminal vertex $v$, and a similar statement holds for $V \in \Pi\left(\mathcal{G}_{2}\right)$. With such $W$ and $V$, the outer homomorphism with representative $f: \pi_{1}\left(\mathcal{G}_{1}, v\right) \rightarrow \pi_{1}\left(\mathcal{G}_{2}, w\right)$ 
is identified with the outer homomorphism with representative $a d_{V} \circ f \circ a d_{W}^{-1}$ : $\pi_{1}\left(\mathcal{G}_{1}, v^{\prime}\right) \rightarrow \pi_{1}\left(\mathcal{G}_{2}, w^{\prime}\right)$.

For any representative homomorphism $f: \pi_{1}\left(\mathcal{G}_{1}, v\right) \rightarrow \pi_{1}\left(\mathcal{G}_{2}, w\right)$, we thus suppress basepoints and denote the corresponding outer homomorphism simply by $\widehat{f}: \pi_{1} \mathcal{G}_{1} \rightarrow \pi_{1} \mathcal{G}_{2}$. If $\mathcal{G}=\mathcal{G}_{1}=\mathcal{G}_{2}, v=w$ and $v^{\prime}=w^{\prime}$ in the discussion above, the groups $\operatorname{Out}\left(\pi_{1}(\mathcal{G}, v)\right)$ and $\operatorname{Out}\left(\pi_{1}\left(\mathcal{G}, v^{\prime}\right)\right)$ are thus canonically identified, and we denote this group by $\operatorname{Out}\left(\pi_{1} \mathcal{G}\right)$.

\section{Isomorphisms of graphs of groups}

4.1. Definition. A graph of groups isomorphism $H: \mathcal{G}_{1} \rightarrow \mathcal{G}_{2}$ is a quadruple of the form

$$
H=\left(H_{\Gamma},\left(H_{v}\right)_{v \in V\left(\mathcal{G}_{1}\right)},\left(H_{e}\right)_{e \in E\left(\mathcal{G}_{1}\right)},(\delta(e))_{e \in E\left(\mathcal{G}_{1}\right)}\right)
$$

where $H_{\Gamma}: \Gamma\left(\mathcal{G}_{1}\right) \rightarrow \Gamma\left(\mathcal{G}_{2}\right)$ is a graph isomorphism, and each $H_{v}: G_{v} \rightarrow G_{H_{\Gamma}(v)}$ and $H_{e}=H_{\bar{e}}: G_{e} \rightarrow G_{H_{\Gamma}(e)}$ is a group isomorphism. (In order to avoid double indices we will often write $H(e)$ and $H(v)$ instead of $H_{\Gamma}(e)$ or $H_{\Gamma}(v)$.) Moreover, $\delta(e) \in G_{\tau(H(e))}$ and (with $a d_{x}$ as defined in $\S 1$ ),

$$
H_{\tau(e)} f_{e}=a d_{\delta(e)} f_{H(e)} H_{e} .
$$

Note. This definition agrees with the restriction to 1-dimensional complexes of the definition by Haefliger $[\mathrm{H}]$ for isomorphisms of complexes of groups. It is a special case of the more general definition of morphism of graph of groups in Bass $[\mathrm{B}]$.

4.2. A graph of groups isomorphism $H: \mathcal{G}_{1} \rightarrow \mathcal{G}_{2}$ induces isomorphisms $H_{*}$ : $\Pi\left(\mathcal{G}_{1}\right) \rightarrow \Pi\left(\mathcal{G}_{2}\right)$ and $H_{* v}: \pi_{1}\left(\mathcal{G}_{1}, v\right) \rightarrow \pi_{1}\left(\mathcal{G}_{2}, H_{\Gamma}(v)\right)$, defined on generators by

$$
\begin{aligned}
H_{*}(r) & =H_{w}(r) \quad \text { for } \quad w \in V(\mathcal{G}), r \in G_{w}, \quad \text { and } \\
H_{*}\left(t_{e}\right) & =\delta(\bar{e}) t_{H(e)} \delta(e)^{-1} .
\end{aligned}
$$

We denote by $\widehat{H}: \pi_{1} \mathcal{G}_{1} \rightarrow \pi_{1} \mathcal{G}_{2}$ the outer isomorphism induced by $H_{* v}$, where $\widehat{H}$ does not depend on the choice of $v \in V\left(\mathcal{G}_{1}\right)$, see 3.11 .

4.3. The composition of two graph of groups isomorphisms $H^{\prime}: \mathcal{G}_{1} \rightarrow \mathcal{G}_{2}$ and $H^{\prime \prime}: \mathcal{G}_{2} \rightarrow \mathcal{G}_{3}$ is a graph of groups isomorphism $H: \mathcal{G}_{1} \rightarrow \mathcal{G}_{3}$ which satisfies $\widehat{H}=\widehat{H^{\prime \prime}} \widehat{H^{\prime}}$ and $H_{*}=H_{*}^{\prime \prime} H_{*}^{\prime}$. To be precise, $H$ is given by $H_{\Gamma}=H_{\Gamma}^{\prime \prime} H_{\Gamma}^{\prime}$, $H_{v}=H_{H_{\Gamma}^{\prime}(v)}^{\prime \prime} H_{v}^{\prime}, H_{e}=H_{H_{\Gamma}^{\prime}(e)}^{\prime \prime} H_{e}^{\prime}$ and $\delta(e)=H_{\tau\left(H^{\prime}(e)\right)}^{\prime \prime}\left(\delta^{\prime}(e)\right) \delta^{\prime \prime}\left(H_{\Gamma}^{\prime}(e)\right)$ if $v \in$ $V\left(\mathcal{G}_{1}\right), e \in E\left(\mathcal{G}_{1}\right)$.

In particular, for any $H: \mathcal{G}_{1} \rightarrow \mathcal{G}_{2}$ there is an inverse isomorphism $H^{-1}: \mathcal{G}_{2} \rightarrow$ $\mathcal{G}_{1}$ which satisfies $\widehat{H}^{-1}=\widehat{H^{-1}}$ and $H_{*}^{-1}=\left(H^{-1}\right)_{*}$. Moreover $\left(H^{-1}\right)_{H(v)}=H_{v}^{-1}$ and $H_{*}^{-1}\left(t_{H(e)}\right)=H_{\tau(\bar{e})}^{-1}\left(\delta(\bar{e})^{-1}\right) t_{e} H_{\tau(e)}^{-1}(\delta(e))$ for all $v \in V\left(\mathcal{G}_{1}\right), e \in E\left(\mathcal{G}_{1}\right)$. 
4.4. Every graph of groups $\mathcal{G}$ gives rise to a tree $\mathcal{T}_{\mathcal{G}}$ on which $\pi_{1}(\mathcal{G}, v)$ acts (see $[\mathrm{B}, 1.16],[\mathrm{CL} 2, \S 5])$.

Bass-Serre theory is built so that the notions of "equivariantly isomorphic tree actions" and "isomorphic graphs of groups" are essentially equivalent. This is stated precisely in the following Lemmas 4.5, 4.6. (See Bass [B], Corollary 4.5 and Proposition 2.4 for detailed proofs.)

4.5. Lemma. Let $\mathcal{G}_{1}$ and $\mathcal{G}_{2}$ be two graphs of groups with an isomorphism $h$ : $\pi_{1}\left(\mathcal{G}_{1}, v_{1}\right) \rightarrow \pi_{1}\left(\mathcal{G}_{2}, v_{2}\right)$ and a simplicial homeomorphism $\widetilde{H}: \mathcal{T}_{\mathcal{G}_{1}} \rightarrow \mathcal{T}_{\mathcal{G}_{2}}$ which is h-equivariant (i.e. $\widetilde{H}(g \cdot x)=h(g) \cdot \widetilde{H}(x)$ for all $g \in \pi_{1}\left(\mathcal{G}_{1}, v_{1}\right)$ and all $\left.x \in \mathcal{T}_{\mathcal{G}_{1}}\right)$. Then there is a graph of groups isomorphism $H: \mathcal{G}_{1} \rightarrow \mathcal{G}_{2}$ with $\widehat{H}=\hat{h}: \pi_{1} \mathcal{G}_{1} \rightarrow$ $\pi_{1} \mathcal{G}_{2}$.

4.6. Lemma. If $H: \mathcal{G}_{1} \rightarrow \mathcal{G}_{2}$ is a graph of groups isomorphism, with induced isomorphism $h=H_{*_{v}}: \pi_{1}\left(\mathcal{G}_{1}, v\right) \rightarrow \pi_{1}\left(\mathcal{G}_{2}, H_{\Gamma}(v)\right)$, then there exists an $h$-equivariant simplicial homeomorphism $\widetilde{H}: \mathcal{T}_{\mathcal{G}_{1}} \rightarrow \mathcal{T}_{\mathcal{G}_{2}}$.

\section{Dehn twists}

5.1. Definition. [CL2, §6] A Dehn twist $D=D\left(\mathcal{G},\left(z_{e}\right)_{e \in E(\mathcal{G})}\right)$ consists of a graph of groups $\mathcal{G}$ and a family of elements $\left(z_{e}\right)_{e \in E(\mathcal{G})}$ with $z_{e} \in \operatorname{Center}\left(G_{e}\right)$ and $z_{\bar{e}}=z_{e}^{-1}$. (" $D$ is based on $\mathcal{G}$ with twistors $z_{e}$ "). This determines an automorphism $D_{*}: \Pi(\mathcal{G}) \rightarrow \Pi(\mathcal{G})$ given on the generators as follows:

$$
\left.D_{*}\right|_{G_{v}}=\mathrm{id}, \quad D_{*}\left(t_{e}\right)=t_{e} f_{e}\left(z_{e}\right) \quad(v \in V(\mathcal{G}), \quad e \in E(\mathcal{G})) .
$$

The automorphism $D_{*}$ restricts to an automorphism $D_{v}: \pi_{1}(\mathcal{G}, v) \rightarrow \pi_{1}(\mathcal{G}, v)$ for every $v \in V(\mathcal{G})$ and hence defines (see 3.11) an outer automorphism $\widehat{D} \in \operatorname{Out}\left(\pi_{1} \mathcal{G}\right)$.

5.2. For any Dehn twist $D=D\left(\mathcal{G},\left(z_{e}\right)_{e \in E(\mathcal{G})}\right)$ and any connected word $W \in \Pi(\mathcal{G})$ with initial vertex $v^{\prime}$ and terminal vertex $v$ Definition 5.1 gives

$$
D_{v^{\prime}} a d_{W}=a d_{D_{*}(W)} D_{v}: \pi_{1}(\mathcal{G}, v) \rightarrow \pi_{1}\left(\mathcal{G}, v^{\prime}\right) .
$$

5.3. Lemma. Let $D_{1}=D\left(\mathcal{G}_{1},\left(z_{e}\right)_{e \in E\left(\mathcal{G}_{1}\right)}\right)$ and $D_{2}=D\left(\mathcal{G}_{2},\left(z_{e}\right)_{e \in E\left(\mathcal{G}_{2}\right)}\right)$ be Dehn twists and $H: \mathcal{G}_{1} \rightarrow \mathcal{G}_{2}$ a graph of groups isomorphism which preserves twistors in that

$$
H_{e}\left(z_{e}\right)=z_{H(e)} \quad \text { for all } \quad e \in E\left(\mathcal{G}_{1}\right)
$$


Then: (a) $H_{*} D_{1 *} H_{*}^{-1}=D_{2 *}: \Pi\left(\mathcal{G}_{2}\right) \rightarrow \Pi\left(\mathcal{G}_{2}\right)$.

(b) $\widehat{D_{2}}=\widehat{H} \widehat{D_{1}} \widehat{H}^{-1} \in \operatorname{Out}\left(\pi_{1} \mathcal{G}_{2}\right)$.

Proof. The claim (a) follows from straightforward calculation on the generators of $\Pi\left(\mathcal{G}_{2}\right)$ using the formulas of 4.2 and 4.3. Then (b) follows from (3.11).

5.4. Proposition. Let $\mathcal{G}$ be a graph of groups with the property that (*) for every edge $e$ there is an element $r_{e} \in G_{\tau(e)}$ with

$$
f_{e}\left(G_{e}\right) \cap r_{e} f_{e}\left(G_{e}\right) r_{e}^{-1}=\{1\} .
$$

Then two Dehn twists $D^{\prime}=D\left(\mathcal{G},\left(z_{e}^{\prime}\right)_{e \in E(\mathcal{G})}\right), D^{\prime \prime}=D\left(\mathcal{G},\left(z_{e}^{\prime \prime}\right)_{e \in E(\mathcal{G})}\right)$ based on $\mathcal{G}$ determine the same outer automorphisms $\widehat{D^{\prime}}=\widehat{D^{\prime \prime}} \in \operatorname{Out}\left(\pi_{1} \mathcal{G}\right)$ if and only if $z_{e}^{\prime}=z_{e}^{\prime \prime}$ for all $e \in E(\mathcal{G})$.

Proof. Suppose $\widehat{D^{\prime}}=\widehat{D^{\prime \prime}}$. For every edge $e \in E(\mathcal{G})$ we consider the element $w_{e}=t_{e} r_{e} t_{\bar{e}} r_{\bar{e}} \in \pi_{1}(\mathcal{G}, v)$, where $v=\tau(\bar{e})$. We compute

$$
\begin{aligned}
& D_{*}^{\prime}\left(w_{e}\right)=t_{e} f_{e}\left(z_{e}^{\prime}\right) r_{e} f_{e}\left(z_{e}^{\prime}\right)^{-1} t_{\bar{e}} r_{\bar{e}} \quad \text { and } \\
& D_{*}^{\prime \prime}\left(w_{e}\right)=t_{e} f_{e}\left(z_{e}^{\prime \prime}\right) r_{e} f_{e}\left(z_{e}^{\prime \prime}\right)^{-1} t_{\bar{e}} r_{\bar{e}} .
\end{aligned}
$$

Since $\widehat{D^{\prime}}=\widehat{D^{\prime \prime}}$, these words represent conjugate elements in $\pi_{1}(\mathcal{G}, v)$. From 3.9 it follows that there is an element $s \in G_{v}$ such that $D_{*}^{\prime}\left(w_{e}\right)=s D_{*}^{\prime \prime}\left(w_{e}\right) s^{-1}$. Then 3.6 implies that there exist elements $h_{1}, h_{2} \in G_{e}$ with

(a) $f_{\bar{e}}\left(h_{1}\right)^{-1}=s$,

(b) $f_{e}\left(h_{1}\right) f_{e}\left(z_{e}^{\prime}\right) r_{e} f_{e}\left(z_{e}^{\prime}\right)^{-1} f_{e}\left(h_{2}\right)^{-1}=f_{e}\left(z_{e}^{\prime \prime}\right) r_{e} f_{e}\left(z_{e}^{\prime \prime}\right)^{-1}$, and

(c) $f_{\bar{e}}\left(h_{2}\right) r_{\bar{e}}=r_{\bar{e}} s^{-1}$.

From (a) and (c), $r_{\bar{e}} f_{\bar{e}}\left(h_{1}\right) r_{\bar{e}}^{-1}=f_{\bar{e}}\left(h_{2}\right)$. So $\left(^{*}\right)$ implies that $h_{1}=h_{2}=1$. Then (b) and (*) imply that $z_{e^{\prime}}^{-1} z_{e}^{\prime \prime}=1$.

The converse implication is obvious.

5.5. An alternative viewpoint, which we will not adopt in this paper, is to consider a Dehn twist $D=D\left(\mathcal{G},\left(z_{e}\right)_{e \in E(\mathcal{G})}\right)$ as a graph of groups automorphism $\mathcal{D}: \mathcal{G} \rightarrow$ $\mathcal{G}$, with identity map for the graph automorphism $\mathcal{D}_{\Gamma}$ and identity maps for all the group automorphisms $\mathcal{D}_{v}$ and $\mathcal{D}_{e}$. Furthermore the elements $\delta(e)$ are chosen to satisfy $\delta(\bar{e}) t_{e} \delta(e)^{-1}=t_{e} f_{e}\left(z_{e}\right)$ for all $e \in E(\mathcal{G})$. (This can be achieved, for example, if one chooses a representative $e^{+}$from each set $\{e, \bar{e}\}$ and defines $\delta\left(e^{+}\right)=$ $f_{e^{+}}\left(z_{e^{+}}\right)^{-1}$ and $\left.\delta\left(\bar{e}^{+}\right)=1\right)$. It follows directly from the definitions in 4.2 and 5.1 that this graph of groups automorphism induces the same automorphisms as $D$ on $\Pi(\mathcal{G})$ and on $\pi_{1}(\mathcal{G}, v)$, for any $v \in V(\mathcal{G})$. 


\section{Efficient Dehn twists}

6.1. General assumption. For the rest of the paper we always assume that for any graph of groups $\mathcal{G}$ the fundamental group $\pi_{1} \mathcal{G}$ is a free group of finite rank $n \geq 2$. We remind the reader that the graph $\Gamma(\mathcal{G})$ is always finite and connected.

6.2. Definition. A Dehn twist $D=D\left(\mathcal{G},\left(z_{e}\right)_{e \in E(\mathcal{G})}\right)$ is called efficient (called proper with all twistors non-trivial in [CL2, §13]) if the following conditions hold:

(i) $\mathcal{G}$ is minimal: There is no vertex $v$ of valence 1 with $v=\tau(e)$ and surjective edge map $f_{e}: G_{e} \rightarrow G_{v}$.

(ii) There is no invisible vertex: There is no vertex $v$ of valence 2 with $v=$ $\tau\left(e_{1}\right)=\tau\left(e_{2}\right)\left(e_{1} \neq e_{2}\right)$ such that both edge maps $f_{e_{i}}: G_{e_{i}} \rightarrow G_{v}, i=1,2$, are surjective.

(iii) There are no unused edges: For every edge $e$ the twistor is non-trivial: $1 \neq z_{e} \in \operatorname{center}\left(G_{e}\right)$. In particular one has $G_{e} \cong \mathbb{Z}$ for all edges $e \in E(\mathcal{G})$.

(iv) There are no proper powers: If $r^{p} \in f_{e}\left(G_{e}\right)$ and $p \neq 0$ then $r \in f_{e}\left(G_{e}\right)$.

(v) If $v=\tau\left(e_{1}\right)=\tau\left(e_{2}\right)$ then the edges $e_{1}$ and $e_{2}$ are not positively bonded: There are no positive powers $m, n$ such that $f_{e_{1}}\left(z_{e_{1}}^{m}\right)$ is conjugate to $f_{e_{2}}\left(z_{e_{2}}^{n}\right)$ in $G_{v}$.

6.3. Remark. Conditions (iii) and (v) imply that:

(vi) There are no conjugate triples: There is no vertex $v=\tau\left(e_{1}\right)=\tau\left(e_{2}\right)=$ $\tau\left(e_{3}\right)$, where $e_{1}, e_{2}, e_{3}$ are distinct edges, such that there exist non-trivial $y_{i} \in G_{e_{i}}(i=1,2,3)$ with $f_{e_{1}}\left(y_{1}\right), f_{e_{2}}\left(y_{2}\right)$ and $f_{e_{3}}\left(y_{3}\right)$ all conjugate in $G_{v}$.

Conditions (iii), (iv) and (vi) assert that $\mathcal{G}$ is a very small graph of groups, as defined in [CL2].

6.4. Lemma. Let $D=D\left(\mathcal{G},\left(z_{e}\right)_{e \in E(\mathcal{G})}\right)$ be an efficient Dehn twist. Then every vertex group $G_{v}$ of $\mathcal{G}$ has rank at least 2. In particular $\mathcal{G}$ satisfies the condition (*) in Proposition 5.4 .

Proof. From 6.2 (iii) and the assumption in 3.1 that $\Gamma(\mathcal{G})$ is connected it follows that every vertex group has rank at least 1 . If for some vertex $v$ of $\Gamma(\mathcal{G})$ one has $G_{v} \cong \mathbb{Z}$, then it follows from 6.2 (iv) that $f_{e}$ is surjective for all edges $e$ with $\tau(e)=v$. Hence, by 6.2 (i) and (ii), there are at least 3 distinct such edges. By 6.2 (iii) each has non-trivial twistor. But then at least 2 of them are positively bonded, in contradiction to $6.2(\mathrm{v})$. Hence every $G_{v}$ has rank at least 2 .

By 6.1 and 6.2 (iii), (iv) the group $f_{e}\left(G_{e}\right)$ is a maximal cyclic subgroup in $G_{\tau(e)}$. But then we can pick any $r_{e} \in G_{\tau(e)}-f_{e}\left(G_{e}\right)$ to satisfy the condition (*) in Proposition 5.4., since maximal cyclic subgroups of free groups are malnormal. This follows easily from considering the standard free action of the free group on a simplicial tree and the minimal subtree (a line !) fixed by the maximal cyclic 
subgroup.

6.5. Lemma. Let $D=D\left(\mathcal{G},\left(z_{e}\right)_{e \in E(\mathcal{G})}\right)$ be an efficient Dehn twist. Let $W=$ $r_{0} t_{1} r_{1} \ldots t_{q} r_{q} \in \Pi(\mathcal{G})$ be a reduced word of length $q$. Then $D_{*}(W)=W$ if and only if $q=0$.

Proof. By definition, words of length 0 are fixed by $D_{*}$. To see that words of greater length are not fixed, start by choosing as generator of the cyclic group $G_{e}$, for each edge $e$, the element $a_{e}$ such that $a_{e}^{n(e)}=z_{e}$ with $n(e)>0$. Since $z_{\bar{e}}=z_{e}{ }^{-1}$ this choice dictates that $n(e)=n(\bar{e})$ and $a_{\bar{e}}=a_{e}{ }^{-1}$. In the word $W$ let each $t_{j}=t_{e_{j}}$, and set $n\left(e_{j}\right)=n_{j}$ and $a_{j}=a_{e_{j}}$. Let $x_{j}=f_{e_{j}}\left(z_{e_{j}}\right)=f_{e_{j}}\left(a_{j}^{n_{j}}\right)$

We claim that: $W=D_{*}(W)$ with $q>0$ would imply

$$
1=r_{q}^{-1} f_{e_{q}}\left(a_{q}^{n_{1}+n_{2}+\cdots+n_{q}}\right) r_{q}
$$

This is impossible since in an efficient graph of groups there are no unused edges, so that $a_{q} \neq 1$ and each $n_{j}>0$.

When $q=1, W^{-1} D_{*}(W)=\left(r_{0} t_{1} r_{1}\right)^{-1}\left(r_{0} t_{1} x_{1} r_{1}\right)$, and the claim is immediate. When $q>1$, note that $D_{*}(W)$ is reduced, since $W$ is reduced. So in the following equation with non-reduced right-hand side,

$$
1=W^{-1} D_{*}(W)=r_{q}^{-1} t_{q}^{-1} \ldots r_{2}^{-1}\left(t_{2}^{-1} r_{1}^{-1} x_{1} r_{1} t_{2}\right) x_{2} r_{2} \ldots t_{q} x_{q} r_{q},
$$

we must have $r_{1}{ }^{-1} x_{1} r_{1}=r_{1}^{-1} f_{e_{1}}\left(a_{e_{1}}^{n_{1}}\right) r_{1} \in f_{\bar{e}_{2}}\left(G_{\bar{e}_{2}}\right)$ and $\tau\left(e_{1}\right)=\tau\left(\bar{e}_{2}\right), r_{1} \in$ $G_{\tau\left(e_{2}\right)}$. Since there are no proper powers by $6.2(\mathrm{iv}), r_{1}^{-1} f_{e_{1}}\left(a_{e_{1}}\right) r_{1}$ itself belongs to $f_{\bar{e}_{2}}\left(G_{\bar{e}_{2}}\right)$. Thus it is a is a power of $f_{\bar{e}_{2}}\left(a_{\bar{e}_{2}}\right)$. But since $r_{1}^{-1} f_{e_{1}}\left(G_{e_{1}}\right) r_{1}$ also contains no proper powers, a symmetric argument gives

$$
r_{1}^{-1} f_{e_{1}}\left(a_{e_{1}}\right) r_{1}=\left(f_{\bar{e}_{2}}\left(a_{\bar{e}_{2}}\right)\right)^{ \pm 1}
$$

By $6.2(\mathrm{v})$, the edges $e_{1}$ and $\bar{e}_{2}$ are not positively bonded, so the exponent sign above is negative. Since $a_{\bar{e}_{2}}=a_{e_{2}}^{-1}$ we conclude (using 3.2) that

$$
\begin{aligned}
1=W^{-1} D_{*}(W) & =r_{q}^{-1} t_{q}^{-1} \ldots r_{2}^{-1} f_{e_{2}}\left(a_{e_{2}}^{n_{1}}\right) x_{2} r_{2} \ldots t_{q} x_{q} r_{q} \\
& =r_{q}^{-1} t_{q}^{-1} \ldots r_{2}^{-1} f_{e_{2}}\left(a_{e_{2}}^{n_{1}+n_{2}}\right) r_{2} \ldots t_{q} x_{q} r_{q}
\end{aligned}
$$

Continuing in this fashion, the claim is proved.

6.6. Lemma. Let $D=D\left(\mathcal{G},\left(z_{e}\right)_{e \in E(\mathcal{G})}\right)$ be an efficient Dehn twist, and consider vertices $v, v^{\prime} \in V(\mathcal{G})$. Suppose that $W \in \Pi(\mathcal{G})$ is a connected word with initial vertex $v^{\prime}$ and terminal vertex $v$ such that

$$
D_{v^{\prime}}=a d_{W} D_{v} a d_{W^{-1}}: \pi_{1}\left(\mathcal{G}, v^{\prime}\right) \rightarrow \pi_{1}\left(\mathcal{G}, v^{\prime}\right) .
$$


Then $v=v^{\prime}$ and $W \in G_{v}$.

Proof. Straightforward calculation using 5.2 gives for any $U \in \pi_{1}\left(\mathcal{G}, v^{\prime}\right)$ :

$$
D_{v^{\prime}}(U)=a d_{W}\left(a d_{W^{-1} D_{*}(W)} D_{v}\right) a d_{W^{-1}}(U) .
$$

Thus our hypothesis implies that $a d_{W^{-1} D_{*}(W)}=1 \in \operatorname{Aut}\left(\pi_{1}(\mathcal{G}, v)\right)$. Since the Dehn twist is efficient, $\pi_{1}(\mathcal{G}, v)$ is a free group of rank at least 2 , by 6.3 . Since $W^{-1} D_{*}(W)$ lies in the center of this free group, and hence is trivial, this lemma follows from 6.5 .

\section{Key Observation}

The key observation of this paper, stated in Theorem 6.9 below (roughly the converse of Lemma 5.3), is that if two efficient Dehn twists determine conjugate outer automorphisms, then their graph of groups data are necessarily equal. Thus in an algorithm for conjugacy it is sufficient to check the graph of groups data. The main ingredient in justifying this observation is the Parabolic Orbits Theorem [CL2, 13.2]. For the convenience of the reader, we now describe its content, in a weakened form and with the terminology adapted to the conventions in this paper.

6.7. Consider the Culler-Vogtmann space $C V_{n}$ (also called "outer space") of free actions of $F_{n}$ on metric simplicial $\mathbb{R}$-trees (i.e. on trees $\mathcal{T}_{\mathcal{G}^{\prime}}$ as in 4.4 with $\pi_{1}\left(\mathcal{G}^{\prime}, v\right) \cong$ $F_{n}$ and all edge groups and vertex groups trivial, provided with a $\pi_{1}\left(\mathcal{G}^{\prime}, v\right)$ equivariant length on the edges). An efficient Dehn twist $D=D\left(\mathcal{G},\left(z_{e}\right)_{e \in E(\mathcal{G})}\right)$ defines [CL2, §9] (after having chosen a "marking", i.e. an identification $\pi_{1}(\mathcal{G}, v)=$ $\left.F_{n}\right)$ a simplex $\sigma(\mathcal{G})$ on the boundary of $C V_{n}$, given by all possible lengths on the edges of $\Gamma(\mathcal{G})$. The Parabolic Orbits Theorem then states that under both forward or backward iteration of the induced action of $\widehat{D}$ every point $\left[\mathcal{T}_{\mathcal{G}^{\prime}}\right]$ of $C V_{n}$ converges to a point in $\sigma(\mathcal{G})$ (which can be precisely determined in terms of the translation lengths on $\mathcal{T}_{\mathcal{G}^{\prime}}$ of the twistors of $D$ ). As $\sigma(\mathcal{G})$ determines $\mathcal{T}_{\mathcal{G}}$ (up to $\pi_{1}(\mathcal{G}, v)$-equivariant isomorphisms), one derives as a consequence:

6.8. Corollary. ([CL2, 13.4], adapted version). Let $D_{1}=D\left(\mathcal{G}_{1},\left(z_{e}\right)_{e \in E\left(\mathcal{G}_{1}\right)}\right)$, $D_{2}=D\left(\mathcal{G}_{2},\left(z_{e}\right)_{e \in E\left(\mathcal{G}_{2}\right)}\right)$ be efficient Dehn twists and let $h: \pi_{1}\left(\mathcal{G}_{1}, v\right) \rightarrow \pi_{1}\left(\mathcal{G}_{2}, w\right)$ be an isomorphism with $\widehat{D_{2}}=\hat{h} \widehat{D_{1}} \hat{h}^{-1} \in \operatorname{Out}\left(\pi_{1} \mathcal{G}_{2}\right)$. Then there exists an $h$ equivariant simplicial homeomorphism $\widetilde{H}: \mathcal{T}_{\mathcal{G}_{1}} \rightarrow \mathcal{T}_{\mathcal{G}_{2}}$.

We have now assembled all ingredients necessary to prove Theorem 1.1 of the Introduction:

6.9. Theorem. Let $D_{1}=D\left(\mathcal{G}_{1},\left(z_{e}\right)_{e \in E\left(\mathcal{G}_{1}\right)}\right)$ and $D_{2}=D\left(\mathcal{G}_{2},\left(z_{e}\right)_{e \in E\left(\mathcal{G}_{2}\right)}\right)$ be efficient Dehn twists inducing automorphisms $D_{v}$ and $D_{w}$ of $\pi_{1}\left(\mathcal{G}_{1}, v\right)$ and $\pi_{1}\left(\mathcal{G}_{2}, w\right)$ respectively. Let $h: \pi_{1}\left(\mathcal{G}_{1}, v\right) \rightarrow \pi_{1}\left(\mathcal{G}_{2}, w\right)$ be an isomorphism. 
(a) If $\widehat{D_{2}}=\hat{h} \widehat{D_{1}} \hat{h}^{-1} \in \operatorname{Out}\left(\pi_{1} \mathcal{G}_{2}\right)$ then there exists a graph of groups isomorphism $H: \mathcal{G}_{1} \rightarrow \mathcal{G}_{2}$ with $\widehat{H}=\hat{h}$ and $H_{e}\left(z_{e}\right)=z_{H(e)}$ for all $e \in E\left(\mathcal{G}_{1}\right)$.

(b)If $D_{w}=h D_{v} h^{-1} \in \operatorname{Aut}\left(\pi_{1}\left(\mathcal{G}_{2}, w\right)\right.$ then there exists a graph of groups isomorphism $H: \mathcal{G}_{1} \rightarrow \mathcal{G}_{2}$ with $H_{\Gamma}(v)=w, H_{* v}=h$ and $H_{e}\left(z_{e}\right)=z_{H(e)}$ for all $e \in E\left(\mathcal{G}_{1}\right)$.

Proof. (a) From Corollary 6.8 and Lemma 4.5 it follows that there is a graph of groups isomorphism $H: \mathcal{G}_{1} \rightarrow \mathcal{G}_{2}$ with $\widehat{H}=\hat{h}$. We consider the Dehn twist $D^{\prime}=$ $D\left(\mathcal{G}_{2},\left(H_{e}\left(z_{e}\right)\right)_{H(e) \in E\left(\mathcal{G}_{2}\right)}\right)$. By Lemma 5.3 we have $\widehat{D^{\prime}}=\widehat{H} \widehat{D_{1}} \widehat{H}^{-1} \in \operatorname{Out}\left(\pi_{1} \mathcal{G}_{2}\right)$. Hence, since $\widehat{H}=\hat{h}$, the hypothesis $\widehat{D_{2}}=\hat{h} \widehat{D_{1}} \hat{h}^{-1}$ implies $\widehat{D^{\prime}}=\widehat{D_{2}} \in \operatorname{Out}\left(\pi_{1} \mathcal{G}_{2}\right)$. Thus Lemma 6.4 and Proposition 5.4 prove $H_{e}\left(z_{e}\right)=z_{H(e)}$ for all $e \in E\left(\mathcal{G}_{1}\right)$.

(b) Consider the outer automorphisms $\widehat{D_{1}} \in \operatorname{Out}\left(\pi_{1}\left(\mathcal{G}_{1}\right)\right)$ and $\widehat{D_{2}} \in \operatorname{Out}\left(\pi_{1}\left(\mathcal{G}_{2}\right)\right)$ which are determined by $D_{v}$ and $D_{w}$. By Part (a) there exists a graph of groups isomorphism $H: \mathcal{G}_{1} \rightarrow \mathcal{G}_{2}$ with $\widehat{H}=\hat{h}$ and $H_{e}\left(z_{e}\right)=z_{H(e)}$ for all $e \in E\left(\mathcal{G}_{1}\right)$. By definition $4.2, \widehat{H}=\widehat{H_{* v}}$ where $H_{* v}: \pi_{1}\left(\mathcal{G}_{1}, v\right) \rightarrow \pi_{1}\left(\mathcal{G}_{2}, H_{\Gamma(v)}\right)$. Thus $\widehat{H_{* v}}=\hat{h}$, and by 3.11 there is a connected word $W \in \Pi\left(\mathcal{G}_{2}\right)$ with initial vertex $H_{\Gamma(v)}$ and terminal vertex $w$ such that

$$
H_{* v}=\operatorname{ad}_{W} h
$$

Again, let $D^{\prime}=D\left(\mathcal{G}_{2},\left(H_{e}\left(z_{e}\right)\right)_{H(e) \in E\left(\mathcal{G}_{2}\right)}\right)$. By 5.3 we then have

$$
D_{*}^{\prime}=H_{*} D_{*} H_{*}^{-1}: \Pi\left(\mathcal{G}_{2}\right) \rightarrow \Pi\left(\mathcal{G}_{2}\right)
$$

and hence

$$
\begin{aligned}
D_{H_{\Gamma}(v)}^{\prime} & =H_{* v} D_{v} H_{* v}^{-1} \\
& =\left(H_{* v} h^{-1}\right) D_{w}\left(h H_{* v}^{-1}\right) \\
& =\operatorname{ad}_{W} D_{w} \operatorname{ad}_{W^{-1}}: \pi_{1}\left(\mathcal{G}_{2}, H_{\Gamma}(v)\right) \rightarrow \pi_{1}\left(\mathcal{G}_{2}, H_{\Gamma}(v)\right)
\end{aligned}
$$

Thus by Lemma 6.6, $H_{\Gamma}(v)=w$ and $W \in G_{w}$. Since $H_{* v}=a d_{W} h: \pi_{1}\left(\mathcal{G}_{1}, v\right) \rightarrow$ $\pi_{1}\left(\mathcal{G}_{2}, w\right)$ we may now define a new graph of groups isomorphism $K: \mathcal{G}_{1} \rightarrow \mathcal{G}_{2}$ with the desired properties by letting $K$ be identical with $H$, except that, for edges $e \in E\left(\mathcal{G}_{1}\right)$ with $\tau(\bar{e})=v$, we have $\delta_{K}(e)=\operatorname{ad}_{W^{-1}} \delta_{H}(e)$.

\section{Centralizer, fixed subgroup and index of an efficient Dehn twist}

In this section we derive some properties of efficient Dehn twists which follow easily from the material presented in the previous sections. Notice that the subsequent $\S 8$ can be read independently from this section. 
7.1. Proposition. (a) Let $D=D\left(\mathcal{G},\left(z_{e}\right)_{e \in E(\mathcal{G})}\right)$ be an efficient Dehn twist with induced outer automorphism $\widehat{D} \in \operatorname{Out}\left(\pi_{1}(\mathcal{G})\right)$. Then the centralizer $C(\widehat{D})$ of $\widehat{D}$ in $\operatorname{Out}\left(\pi_{1}(\mathcal{G})\right)$ is given by

$$
C(\widehat{D})=\left\{\widehat{H} \mid H: \mathcal{G} \stackrel{\cong}{\longrightarrow} \mathcal{G}, \quad H_{e}\left(z_{e}\right)=z_{H(e)} \quad \text { for all } e \in E(\mathcal{G})\right\} .
$$

(b) If $v \in V(\mathcal{G})$ the centralizer of the automorphism $D_{v}$ in $A u t\left(\pi_{1}(\mathcal{G}, v)\right)$ is given by

$$
C\left(D_{v}\right)=\left\{H_{* v} \mid H: \mathcal{G} \stackrel{\cong}{\longrightarrow}, H_{e}\left(z_{e}\right)=z_{H(e)} \text { for all } e \in E(\mathcal{G}), H_{\Gamma}(v)=v\right\} .
$$

Proof. In both (a) and (b) it follows from the special case $D_{1}=D_{2}$ in Lemma 5.3 that the right hand side is contained in the left hand side. The opposite inclusions follow similarily from Theorem 6.9 (with $v=w$ in case (b)).

7.2. Proposition. If $D=D\left(\mathcal{G},\left(z_{e}\right)_{e \in E(\mathcal{G})}\right)$ is an efficient Dehn twist and $v$ is a vertex of $\mathcal{G}$, then Fix $\left(D_{v}\right)=G_{v}$.

Proof. This is an immediate corollary of 6.5 .

7.3. For any graph of groups $\mathcal{G}$, with finitely generated fundamental group isomorphic to a free group $F_{n}$ and finitely generated vertex and edge groups, an elementary Mayer-Vietoris argument in group homology (pointed out to us by M. Bridson) gives the formula

$$
r k\left(\pi_{1}(\mathcal{G})\right)=\sum_{v \in V(\mathcal{G})} r k\left(G_{v}\right)-\sum_{e \in E^{+}(\mathcal{G})} r k\left(G_{e}\right)+r k\left(\pi_{1}(\Gamma(\mathcal{G})),\right.
$$

where $E^{+}(\mathcal{G})$ consists of one "positively oriented" edge from each pair of oppositely oriented edges in $E(\mathcal{G})$ - so that $\# E^{+}(\mathcal{G})$ is the number of geometric edges in the graph. But note that $r k\left(\pi_{1}(\Gamma(\mathcal{G}))=1-\chi(\Gamma(\mathcal{G}))=1+\# E^{+}(\mathcal{G})-\# V(\mathcal{G})\right.$. Thus, if every group $G_{e}$ is isomorphic to $\mathbb{Z}$, we obtain:

$$
\begin{aligned}
r k\left(\pi_{1}(\mathcal{G})\right)-1 & =\sum_{v \in V(\mathcal{G})} r k\left(G_{v}\right)-\# E^{+}(\mathcal{G})+\left(1+\# E^{+}(\mathcal{G})-\# V(\mathcal{G})\right)-1 \\
& =\sum_{v \in V(\mathcal{G})}\left(r k\left(G_{v}\right)-1\right)
\end{aligned}
$$

7.4. In [GJLL] the index of a free group automorphism $\Phi$ is defined by

$$
\operatorname{ind}(\Phi)=r k(\operatorname{Fix}(\Phi))+a(\Phi) / 2-1
$$


where $a(\Phi)$ denotes the number of equivalence classes of attracting infinite fixed words of $\Phi$, see [GJLL] or [CL1]. The main theorem of [GJLL] asserts that if $\Phi_{1}, \Phi_{2}, \cdots \Phi_{q}$ are automorphisms of a free group $F_{n}$ of rank $n$ which determine the same outer automorphism class $\phi$ and are pairwise non-similar (i.e., $\Phi_{i} \neq$ $a d_{u} \Phi_{j} a d_{u^{-1}}$ for any $u \in F_{n}$ if $\left.i \neq j\right)$, then one has

$$
\sum_{i=1, \ldots q} i n d\left(\Phi_{i}\right) \leq n-1
$$

The index $\operatorname{ind}(\phi)$ of the outer automorphism $\phi$ is defined to be the maximum of the left hand side, taken over all possible sets of pairwise non-similar automorphisms $\left\{\Phi_{1}, \Phi_{2}, \ldots, \Phi_{q}\right\}$.

7.5. Consider vertices $v, w \in V(\mathcal{G})$ and a connected word $W \in \Pi(\mathcal{G})$ with initial vertex $w$ and terminal vertex $v$. Then 5.2 gives

$$
a d_{W} D_{v} a d_{W^{-1}}=a d_{W D_{*}\left(W^{-1}\right)} D_{w}: \pi_{1}(\mathcal{G}, w) \rightarrow \pi_{1}(\mathcal{G}, w)
$$

and, as $W D_{*}\left(W^{-1}\right) \in \pi_{1}(\mathcal{G}, w)$, this automorphism also represents the outer automorphism $\hat{D}$. Furthermore, by 7.2 , one has

$$
\operatorname{Fix}\left(a d_{W} D_{v} a d_{W^{-1}}\right)=a d_{W}\left(\operatorname{Fix}\left(D_{v}\right)\right)=a d_{W}\left(G_{v}\right)
$$

and hence, by Lemma $6.4, r k\left(\right.$ Fix $\left(a d_{W} D_{v} a d_{W-1}\right) \geq 2$.

7.6. Lemma. Let $W, W^{\prime} \in \Pi(\mathcal{G})$ be connected words with initial vertex $w$ and terminal vertices $v$ and $v^{\prime}$ respectively. Then the automorphism $a d_{W} D_{v} a d_{W-1}$ and $a d_{W^{\prime}} D_{v^{\prime}} a d_{W^{\prime-1}}$ of $\pi_{1}(\mathcal{G}, w)$ are similar if and only if $v=v^{\prime}$.

Proof. If $v=v^{\prime}$ then $W^{\prime} W^{-1} \in \pi_{1}(\mathcal{G}, w)$ and $a d_{W^{\prime} W^{-1}}\left(a d_{W} D_{v} a d_{W^{-1}}\right) a d_{W W^{\prime-1}}$ $=a d_{W^{\prime}} D_{v^{\prime}} a d_{W^{\prime-1}}$. Hence the two automorphisms are similar.

On the other hand, for any $W^{\prime \prime} \in \pi_{1}(\mathcal{G}, w)$ the equation

$$
a d_{W^{\prime \prime}}\left(a d_{W} D_{v} a d_{W^{-1}}\right) a d_{W^{\prime \prime-1}}=a d_{W^{\prime}} D_{v^{\prime}} a d_{W^{\prime-1}}
$$

is equivalent to

$$
a d_{W^{\prime-1} W^{\prime \prime} W} D_{v} a d_{W^{-1} W^{\prime \prime-1} W^{\prime}}=D_{v^{\prime}}
$$

Thus Lemma 6.6 implies $v=v^{\prime}$.

As a direct consequence of $7.2-7.6$ we obtain:

7.7. Corollary. Let $D=D\left(\mathcal{G},\left(z_{e}\right)_{e \in E(\mathcal{G})}\right)$ be an efficient Dehn twist and let $w \in V(\mathcal{G})$ be any vertex. We have: 
(a) $\operatorname{ind}(\hat{D})=r k\left(\pi_{1}(\mathcal{G})\right)-1$.

(b) Every representative $\Phi \in \operatorname{Aut}\left(\pi_{1}(\mathcal{G}, w)\right)$ of $\hat{D}$ with ind $(\Phi)>0$ is similar to $a d_{W} D_{v} a d_{W^{-1}}$ for some vertex $v \in V(\mathcal{G})$ and any connected word $W$ with initial vertex $w$ and terminal vertex $v$.

(c) None of the $D_{v}$ has attracting infinite fixed words: $a\left(D_{v}\right)=0$ for all $v \in V(\mathcal{G})$.

Notice also that a particular interesting case arises if $\Gamma(\mathcal{G})$ has only one vertex $v$, as then $\operatorname{rank} \operatorname{Fix}\left(D_{v}\right)=n$. Automorphisms $\Phi \in \operatorname{Aut}\left(F_{n}\right)$ with $\operatorname{rank}(\operatorname{Fix}(\Phi))=n$ have been investigated in [CT]; it follows from [L2] or [CT] that they are in fact all Dehn twists with a single vertex. Hence our solution to the conjugacy problem applies also to this class of automorphisms.

We conclude this section by restating our structural result in Theorem 6.9 in a language which comes close to a "normal form" for Dehn twists:

7.8. Assume we are given a finite connected graph $\Gamma$ and two finite families of integers $(r(v))_{v \in V(\Gamma)}$ and $(n(e))_{e \in E(\Gamma)}$, all of which elements satisfy $r(v) \geq 2$, $n(e) \geq 1$ and $n(e)=n(\bar{e})$. Furthermore let $\left(\alpha_{e}\right)_{e \in E(\Gamma)}$ be a family of elements $\alpha_{e} \in F_{r(\tau(e))}$, where $F_{n}$ denotes the (standard copy of a) free group of rank $n \in \mathbb{N}$.

We consider the graph of groups $\mathcal{G}$ given by $\Gamma(\mathcal{G})=\Gamma, \gamma_{v}: G_{v} \stackrel{\cong}{\longrightarrow} F_{r(v)}$, $\gamma_{e}: G_{e} \stackrel{\cong}{\longrightarrow} \mathbb{Z}$ and $f_{e}\left(a_{e}\right)=\gamma_{v}^{-1}\left(\alpha_{e}\right)$, with $a_{e}$ defined through $a_{e}=\gamma_{e}^{-1}(1)$ (for all $v \in V(\Gamma), e \in E(\Gamma))$. We assume that $\gamma_{\bar{e}}=-\gamma_{e}$.

Let $D$ be the Dehn twist based on $\mathcal{G}$ with twistors $a_{e}^{n(e)}$. If $D$ is efficient, then we call the data set

$$
\Delta=\left(\Gamma,(r(v))_{v \in V(\Gamma)},(n(e))_{e \in E(\Gamma)},\left(\alpha_{e}\right)_{e \in E(\Gamma)}\right)
$$

efficient and write $D=D(\Delta), \mathcal{G}=\mathcal{G}(\Delta)$. Obviously we can associate to every efficient Dehn twist $D$ an efficient data set $\Delta$ with $D(\Delta)=D$.

7.9. Corollary. (a) Two Dehn twists $D=D(\Delta)$ and $D^{\prime}=D\left(\Delta^{\prime}\right)$, defined by efficient data sets $\Delta=\left(\Gamma(r(v))_{v \in V(\Gamma)},(n(e))_{e \in E(\Gamma)},\left(\alpha_{e}\right)_{e \in E(\Gamma)}\right)$ and $\Delta^{\prime}=$ $\left(\Gamma^{\prime},(r(v))_{v \in V\left(\Gamma^{\prime}\right)},(n(e))_{e \in E\left(\Gamma^{\prime}\right)},\left(\alpha_{e}\right)_{e \in E\left(\Gamma^{\prime}\right.}\right)$, determine conjugate outer automorphisms if and only if there is a graph isomorphism $H: \Gamma \rightarrow \Gamma^{\prime}$ and automorphisms $\Phi_{v}: F_{r(v)} \rightarrow F_{r(v)}$ for each $v \in V(\Gamma)$, which satisfy

(i) $r(v)=r(H(v))$ for all $v \in V(\Gamma)$,

(ii) $n(e)=n(H(e))$ for all $e \in E(\Gamma)$, and

(iii) $\left[\alpha_{H(e)}\right]=\Phi_{r(e)}\left[\alpha_{e}\right]$ for all $e \in E(\Gamma)$ (where brackets denote the conjugacy class).

(b) For vertices $w \in V(\Gamma), w^{\prime} \in V\left(\Gamma^{\prime}\right)$ the automorphisms $D_{w}$ and $D_{w^{\prime}}^{\prime}$ determined by $D$ and $D^{\prime}$ as in (a) are conjugate if and only if there are $H$ and $\Phi_{v}$ as above, which satisfy the conditions (i) - (iii), and furthermore 
(iv) $H(w)=w^{\prime}$.

Proof. This is a direct consequence of Theorem 6.9.

\section{The algorithm}

In this section we describe a complete algorithm which decides whether or not two given Dehn twists define conjugate outer automorphisms. The algorithm consists of two parts: The first part (Algorithm I) is an algorithm which, given an arbitrary Dehn twist $D$, constructs an efficient Dehn twist $D^{\prime}$ with induced outer automorphism $\widehat{D^{\prime}}$ conjugate to $\widehat{D}$. The second part (Algorithm II) checks whether two given efficient Dehn twists $D_{1}, D_{2}$ define conjugate outer automorphisms.

8.1. Throughout this section we assume that the data for $D=D\left(\mathcal{G},\left(z_{e}\right)_{e \in E(\mathcal{G})}\right)$ are given combinatorially as follows: For every vertex group $G_{v}$ and edge group $G_{e}$ one has specified some basis and has given the injections $f_{e}$ in terms of these bases. If the twistor $z_{e} \neq 1$ then $G_{e}$ is infinite cyclic, since it has non-trivial center, and we choose the generator $a_{e}$ so that $z_{e}=a_{e}{ }^{n(e)}$ with $n(e)>0$. We call the exponent $n(e) \in \mathbb{Z}$ the twist exponent. If $z_{e}=1$ we set $n(e)=0$. This convention implies that $n(e)=n(\bar{e})$ and $a_{\bar{e}}=a_{e}^{-1}$, since $z_{\bar{e}}=z_{e}^{-1}$.

8.2. The Moves. We describe 5 operations by which a given Dehn twist $D=$ $D\left(\mathcal{G},\left(a_{e}^{n(e)}\right)_{e \in E(\mathcal{G})}\right)$ may be changed to a Dehn twist $D^{\prime}=D\left(\mathcal{G}^{\prime},\left(a_{e}^{n(e)}\right)_{e \in E\left(\mathcal{G}^{\prime}\right)}\right)$ with induced automorphism $\widehat{D^{\prime}}$ conjugate to $\widehat{D}$ :

(1) Transition to a proper subgraph: If $\Gamma(\mathcal{G})$ contains a vertex $v$ of valence 1 and if the adjacent edge $e$ has $f_{e}: G_{e} \rightarrow G_{v}$ an isomorphism, then $\mathcal{G}^{\prime}$ is obtained from $\mathcal{G}$ by deleting both $v$ and $e$, with all other data unchanged.

(2) Delete an invisible vertex with negatively bonded edges: If a vertex $v$ of $\Gamma(\mathcal{G})$ is adjacent to precisely 2 edges $e^{\prime}$ and $e^{\prime \prime}$ (both oriented towards $v$ ), and if both edge injections $f_{e^{\prime}}, f_{e^{\prime \prime}}$ are isomorphisms such that $f_{e^{\prime}}\left(a_{e^{\prime}}\right)=f_{e^{\prime \prime}}\left(a_{e^{\prime \prime}}^{-1}\right)$, then $\mathcal{G}^{\prime}$ is derived from $\mathcal{G}$ by replacing $e^{\prime}, e^{\prime \prime}$ and $v$ by a single edge $e$ which runs from $\tau\left(\bar{e}^{\prime}\right)$ to $\tau\left(\bar{e}^{\prime \prime}\right)$. Define $G_{e}=G_{v}, f_{e}=f_{\bar{e}^{\prime \prime}} f_{e^{\prime \prime}}^{-1}, f_{\bar{e}}=f_{\bar{e}^{\prime}} f_{e^{\prime}}^{-1}, a_{e}=f_{e^{\prime}}\left(a_{e^{\prime}}\right)$ and $n(e)=n\left(e^{\prime}\right)+n\left(\bar{e}^{\prime \prime}\right)$.

(3) Fold positively bonded edges: Consider two edges $e, e^{\prime}$ with twist exponents $0<n(e) \leq n\left(e^{\prime}\right)$ and common end point $\tau(e)=\tau\left(e^{\prime}\right)=v$, such that $f_{e}\left(a_{e}\right)=$ $r f_{e^{\prime}}\left(a_{e^{\prime}}\right) r^{-1}$ for some $r \in G_{v}$. Replace $e^{\prime}$ by a new edge $e^{\prime \prime}$ which joins $\tau\left(\bar{e}^{\prime}\right)$ to $\tau(\bar{e})$ and define $G_{e^{\prime \prime}}=G_{e^{\prime}}, a_{e^{\prime \prime}}=a_{e^{\prime}}, f_{\bar{e}^{\prime \prime}}=f_{\bar{e}^{\prime}}, f_{e^{\prime \prime}}\left(a_{e^{\prime \prime}}\right)=f_{\bar{e}}\left(a_{e}\right)$ and $n\left(e^{\prime \prime}\right)=n\left(e^{\prime}\right)-n(e)$.

(4) Contract unused edges: If $e$ is an edge with $a_{e}^{n(e)}=1$, then replace $e$ together with its endpoints $\tau(e), \tau(\bar{e})$ by a single vertex $v$ with vertex group an 
amalgamated product

$$
G_{v}=G_{\tau(\bar{e})} \underset{G_{e}}{*} G_{\tau(e)}, \quad \text { if } \quad \tau(e) \neq \tau(\bar{e}),
$$

or an $\mathrm{HNN}-$ extension

$$
G_{v}=\left\langle G_{\tau(e)}, \theta \mid \theta^{-1} f_{\bar{e}}\left(G_{e}\right) \theta=f_{e}\left(G_{e}\right)\right\rangle, \quad \text { if } \quad \tau(e)=\tau(\bar{e}) .
$$

For any edge $e^{\prime}$ pointing towards $\tau(e)$ or $\tau(\bar{e})$ replace $f_{e^{\prime}}$ by the composition of $f_{e^{\prime}}$ with the canonical injection of $G_{\tau(e)}$ or $G_{\tau(\bar{e})}$ into $G_{v}$.

(5) Get rid of proper powers: If the generator $a_{e}$ of an edge group $G_{e}$ is mapped by $f_{e}$ to a proper power $g^{p} \in G_{\tau(e)}$, then we change $\mathcal{G}$ as follows: We replace $e$ by a new edge $e^{\prime}$ with same endpoints as $e$ and $G_{e^{\prime}}=\langle g\rangle, a_{e^{\prime}}=g, n\left(e^{\prime}\right)=p n(e)$. We replace the vertex group $G_{\tau(\bar{e})}$ by a new group

$$
G_{\tau\left(\bar{e}^{\prime}\right)}=G_{\tau(\bar{e})} \underset{G_{e}}{*}\langle g\rangle
$$

and define $f_{e^{\prime}}$ as well as $f_{\bar{e}^{\prime}}$ to be the canonical injections. For any edge $e^{\prime \prime}$ pointing towards $\tau(\bar{e})$ replace $f_{e^{\prime \prime}}$ by the composition of $f_{e^{\prime \prime}}$ with the canonical injection of $G_{\tau(\bar{e})}$ into $G_{\tau\left(\bar{e}^{\prime}\right)}$.

8.3. Lemma. For any of the operations (1) - (5) in Definition 8.2 and proper choices of vertices $w \in V(\mathcal{G}), w^{\prime} \in V\left(\mathcal{G}^{\prime}\right)$ there is an isomorphism between fundamental groups $\rho: \pi_{1}(\mathcal{G}, w) \rightarrow \pi_{1}\left(\mathcal{G}^{\prime}, w^{\prime}\right)$ which satisfies $\widehat{D^{\prime}} \hat{\rho}=\hat{\rho} \widehat{D}$.

Proof. For operation (1) we choose $w \neq v$ and define $w^{\prime}$ to be the corresponding vertex of $\Gamma\left(\mathcal{G}^{\prime}\right)$. The isomorphism $\rho$ is given through replacing every letter in a reduced word of $\pi_{1}(\mathcal{G}, w)$ by the corresponding letter of $\pi_{1}\left(\mathcal{G}^{\prime}, w^{\prime}\right)$. For the other operations we proceed similarily: For (2) we choose $w \neq v$ and define $\rho$ through deleting in every word any occurence of $t_{e^{\prime}}$ or $t_{e^{\prime}}^{-1}$, and through replacing any $t_{e^{\prime \prime}}$ by $t_{e}^{-1}$ (and $t_{e^{\prime \prime}}^{-1}$ by $t_{e}$ ) as well as any $r \in G_{v}$ by $f_{\bar{e}}(r)$, while leaving all other symbols unchanged. For (3) choose $w$ arbitrary and define $\rho$ through replacing any $t_{e^{\prime}}$ by the product $t_{e^{\prime \prime}} t_{e} r$ (and any $t_{\bar{e}^{\prime}}$ by $r^{-1} t_{\bar{e}} t_{\bar{e}^{\prime \prime}}$ ). For (4) let $w \neq \tau(\bar{e}), \tau(e)$ (and thus $w^{\prime} \neq v$ ), and in the amalgamated product case simply delete any $t_{e}$, whereas in the HNN case replace $t_{e}$ by $\theta$ (and similarily with the inverses). For operation (5) we chose $w$ arbitrary and replace $t_{e}$ by $t_{e^{\prime}}$ (and $t_{\bar{e}}$ by $t_{\bar{e}^{\prime}}$ ).

The equation $\widehat{D^{\prime}} \hat{\rho}=\hat{\rho} \widehat{D}$ can be verified directly from these definitions for $\rho$ and from the Definition 5.1.

8.4. Proposition. For any Dehn twist one can iteratively apply the operations (1) - (5) from 8.2 only a finite number of times.

Proof. Notice that the following facts are true for each of the operations (1) - (5): 
(a) The number of edges does not increase when passing from $\mathcal{G}$ to $\mathcal{G}^{\prime}$.

(b) In the free group $\pi_{1}\left(\mathcal{G}^{\prime}, v^{\prime}\right)$ the set of conjugacy classes of edge group generators $\left\{\left[f_{e}\left(a_{e}\right)\right] \mid e \in E\left(\mathcal{G}^{\prime}\right)\right\}$ differs from the set $\left\{\rho\left[f_{e}\left(a_{e}\right)\right] \mid e \in E(\mathcal{G})\right\}$ (for $\rho$ as in 8.3) only in that some classes of the latter may have been deleted or replaced by proper roots.

Hence (1), (2), (4) and (5) can be applied only a finite number of times: The operations (1), (2) and (4) strictly decrease the number of edges in $\mathcal{G}$, whereas $(5)$ replaces some of the $\rho\left[f_{e}\left(a_{e}\right)\right]$ by a proper root. But every conjugacy class in a free group has only finitely many proper roots.

Operation (3) strictly decreases the total number of twist exponents and hence can be applied only finitely many times before, after or between the operations of type (1), (2), (4) or (5).

8.5. Proposition. If none of the operations (1) - (5) can be performed on a given Dehn twist $D$, then $D$ is efficient.

Proof. This follows directly from the definitions.

8.6. Algorithm I. Given any Dehn twist $D$, check whether any of the operations (1) - (5) in 8.2 can be performed. (As $D$ is given as in 8.1 this can be done in finitely many steps.) If so, do the operation; in case more than one is possible, chose any one at random. Then rename the obtained Dehn twist $D^{\prime}$ to $D$ and repeat the procedure. If none of the operations can be performed, stop.

Summarizing $8.3-8.6$ we obtain:

8.7. Corollary. Algorithm I transforms any Dehn twist in finitely many steps into an efficient Dehn twist without changing the conjugacy class of the induced outer automorphism.

Next we present the algorithm for deciding the conjugacy problem for efficient Dehn twists:

8.8. Algorithm II. Given two efficient Dehn twists $D_{1}=D\left(\mathcal{G}_{1},\left(a_{e}^{n(e)}\right)_{e \in E\left(\mathcal{G}_{1}\right)}\right)$ and $D_{2}=D\left(\mathcal{G}_{2},\left(a_{e}^{n(e)}\right)_{e \in E\left(\mathcal{G}_{2}\right)}\right)$, proceed as follows:

(1) Check whether there is a graph isomorphism $H_{\Gamma}: \Gamma\left(\mathcal{G}_{1}\right) \rightarrow \Gamma\left(\mathcal{G}_{2}\right)$ with $\operatorname{rank}\left(G_{v}\right)=\operatorname{rank}\left(G_{H(v)}\right)$ and $n(e)=n\left(H_{\Gamma}(e)\right)$ for all $v \in V\left(\mathcal{G}_{1}\right), e \in E\left(\mathcal{G}_{1}\right)$. If so, list all (finitely many) such graph isomorphisms.

(2) For each $H_{\Gamma}$ listed in (1) and every $v \in V\left(\mathcal{G}_{1}\right)$ check whether there is an isomorphism $H_{v}: G_{v} \rightarrow G_{H(v)}$ such that $H_{v}\left(f_{e}\left(a_{e}\right)\right)$ is conjugate to $f_{H(e)}\left(a_{H(e)}\right)$ for all $e \in E\left(\mathcal{G}_{1}\right)$ with $\tau(e)=v$. This can be done by applying the Whitehead algorithm to the two families of conjugacy classes,

$$
\left(\left[f_{e}\left(a_{e}\right)\right] \mid e \in E\left(\mathcal{G}_{1}\right), \tau(e)=v\right)
$$


in $G_{v}$, and

$$
\left(\left[f_{H(e)}\left(a_{H(e)}\right)\right] \mid e \in E\left(\mathcal{G}_{1}\right), \tau(e)=v\right)
$$

in $G_{H(v)}$, see for example $[\mathrm{H}]$.

8.9. Proposition. Algorithm II decides whether, given two efficient Dehn twists $D_{1}=D\left(\mathcal{G}_{1},\left(a_{e}^{n(e)}\right)_{e \in E\left(\mathcal{G}_{1}\right)}\right)$ and $D_{2}=D\left(\mathcal{G}_{2},\left(a_{e}^{n(e)}\right)_{e \in E\left(\mathcal{G}_{2}\right)}\right)$, there exists a graph of groups isomorphism $H: \mathcal{G}_{1} \rightarrow \mathcal{G}_{2}$ with $H_{e}\left(a_{e}^{n(e)}\right)=a_{H(e)}^{n(H(e))}$ for all $e \in E\left(\mathcal{G}_{1}\right)$.

Proof. If the algorithm finds in step (1) a graph isomorphism $H_{\Gamma}$ and in step (2) isomorphisms $H_{v}$ for all $v \in V\left(\mathcal{G}_{1}\right)$ with the desired properties, then we can complete these data to a graph of groups isomorphism $H: \mathcal{G}_{1} \rightarrow \mathcal{G}_{2}$ as follows: We define $H_{e}$ by $H_{e}\left(a_{e}\right)=a_{H(e)}$ and define the elements $\delta(e)$ to be the elements in $G_{\tau(H(e))}$ which conjugate $H_{\tau(e)}\left(f_{e}\left(a_{e}\right)\right)$ to $f_{H(e)}\left(a_{H(e)}\right)$, which exist by step $(2)$. The equation $\left({ }^{*}\right)$ in 4.1 follows then directly from these definitions. Thus $H$ is a graph of groups isomorphism and from $n(e)=n\left(H_{\Gamma}(e)\right)$ as given through step (1) we obtain $H_{e}\left(a_{e}^{n(e)}\right)=a_{H(e)}^{n(H(e))}$ for all $e \in E\left(\mathcal{G}_{1}\right)$.

Conversely, if a graph of groups isomorphism $H: \mathcal{G}_{1} \rightarrow \mathcal{G}_{2}$ with $H_{e}\left(a_{e}^{n(e)}\right)=$ $a_{H(e)}^{n(H(e))}$ for all $e \in E\left(\mathcal{G}_{1}\right)$ exists, then it explicitly gives a graph isomorphism $H_{\Gamma}$ as in (1) and vertex group isomorphisms $H_{v}$ as in (2).

8.10. Corollary. Algorithm I and Algorithm II together give a solution to the conjugacy problem for outer automorphisms of free groups defined by Dehn twists.

Proof. Let $D_{1}$ and $D_{2}$ be two (not necessarily efficient) Dehn twists, and let $D_{1}^{\prime}$ and $D_{2}^{\prime}$ be the efficient Dehn twists based on, say, $\mathcal{G}_{1}^{\prime}$ and $\mathcal{G}_{2}^{\prime}$, which are obtained from $D_{1}$ and $D_{2}$ by Algorithm I . By Lemma 8.3 the automorphisms $\widehat{D_{1}}$ and $\widehat{D_{2}}$ are conjugate if and only $\widehat{D_{1}^{\prime}}$ and $\widehat{D_{2}^{\prime}}$ are. Algorithm II decides whether there exists a graph of groups isomorphism $H: \mathcal{G}_{1}^{\prime} \rightarrow \mathcal{G}_{2}^{\prime}$ which preserves twistors. If so, $\widehat{D_{1}}$ and $\widehat{D_{2}}$ and hence $\widehat{D_{1}^{\prime}}$ and $\widehat{D_{2}^{\prime}}$ are conjugate, and otherwise they are not: This is precisely the content of Theorem 6.9 (a) .

8.11. Notice that Algorithm II also solves the conjugacy problem for (non-outer) automorphisms of free groups which are given by efficient Dehn twists: By Theorem 6.9 (b) two such automorphisms $D_{v}$ and $D_{v^{\prime}}^{\prime}$ are conjugate if and only if there exists a corresponding graph of groups isomorphism $H$ which preserves twistors and maps $v$ to $v^{\prime}$. But the existence of such an $H$ is detected precisely by Algorithm II. 


\section{Bibliography}

[B] H. Bass, Covering theory for graphs of groups, J.Pure and Applied Algebra 89 (1993), 3-47.

[C] D. E. Cohen, Combinatorial Group Theory: A topological approach, LMS Student Texts 14, Cambridge Univ. Press, 1989.

[CL1] M. M. Cohen and M. Lustig, On the dynamics and the fixed subgroup of a free group automorphism, Inventiones Math. 96 (1989), 613-638.

[CL2] M. M. Cohen and M. Lustig, Very small group actions on R-trees and Dehn twist automorphisms, Topology 34 (1995), 575-617.

[CT] D. J. Collins and E. C. Turner, All automorphisms of free groups with maximal fixed subgroups, Math. Proc. Cambridge Phil. Soc. 119 (1996), 615-630.

[DD] W. Dicks and M. J. Dunwoody, Groups Acting on Graphs, Cambridge Studies in Adv. Math. 17, Cambridge Univ. Press, 1989.

[H] A. H. M. Hoare, Coinitial graphs and Whitehead automorphisms, Canadian. J. of Math. 31 (1979), 112-123.

[GJLL] D. Gaboriau, A. Jäger, G. Levitt and M. Lustig, An index for counting fixed points of automorphisms of free groups, to appear in Duke Math. J.

[KLV] S. Krstic, M. Lustig and K. Vogtmann, An equivariant Whitehead algorithm and conjugacy for roots of Dehn twists, preprint 1997.

[L1] M. Lustig, Prime factorization and conjugacy problem in $\operatorname{Out}\left(F_{n}\right)$, survey preprint, July 1994.

[L2] M. Lustig, The structure of free group automorphisms, in preparation.

[S] J. P. Serre, Trees, Springer-Verlag, 1980.

Marshall M. Cohen

Department of Mathematics

Cornell University, Ithaca

NY 14853, USA

e-mail: marshall@math.cornell.edu
Martin Lustig

Mathematik

Ruhr Universität Bochum

44780 Bochum, Germany

e-mail: Martin.Lustig@rz.ruhr-uni-bochum.de

(Received: February 12, 1996) 